\title{
NEAR FIELD PHOTOTHERMAL ANNEALING FOR RAPID LASER PRINTING OF METALLIC MICRO AND NANO STRUCTURES
}

\author{
Fan Xiao ${ }^{1 *}$, Ting-Hsiang $\mathrm{Wu}^{2}$, and Pei Yu Chiou ${ }^{1}$
}

${ }^{1}$ Department of Mechanical and Aerospace Engineering, University of California at Los Angeles, USA

${ }^{2}$ Department of Electrical Engineering, University of California at Los Angeles, USA

\begin{abstract}
We report on a rapid near field photothermal printing (NPTP) approach for fabricating metallic micro- and nano-structures guided by flexible and transparent polymer molds. The optical energy of nanosecond laser pulse is spatially redistributed by a PDMS phaseshifting mask, resulting in selective gold melting in light intensity enhanced areas. The melted gold migrates to cold areas and forms desired structures after cooled down. Using NPTP, We have demonstrated rapid laser printing of periodic Au nanowire array with a $320 \mathrm{~nm}$ linewidth, nanosphere arrays with $400 \mathrm{~nm}$ in diameter, and non-periodic structures on both glass and PDMS substrate across a $1 \mathrm{~mm}^{2}$ area with few laser pulses.
\end{abstract}

\section{INTRODUCTION}

Metallic micro and nanostructures have wide applications in solar cell harvesting[1], plasmonic devices[2], color filters[3], and biomedical fields such as metallic particle guided gene delivery[4], photothermal therapy[5], and biosensors[6]. Methods for fabricating metallic nanostructures are versatile, ranging from chemical synthesis, electron beam lithography, ion beam lithography, nanoimprint lithography (NIL)[7], and laser induced dewetting processes. Among them, laser induced dewetting process is considered as an economical method for rapid and large area fabrication.

To future guide laser induced dewetting and self-organization processes, two types of methods have been proposed, including using pre-patterned substrates[8], and spatially shaping the projected laser patterns[9, 10]. Linearly polarized pulse laser intrinsically induces spatially periodic energy density distribution and Multi-beam Interference Lithography (MIL) utilizes at least two laser beams to redistribute periodic energy density at the metallic film surface. They both belong to the later type and allow low cost and rapid fabrication of two-dimensional nanowires and nanoparticles on a continuous thin metallic film. Using nanosecond laser pulses, MIL based photothermal annealing techniques are capable of direct laser printing of periodic metallic nanostructures from thin metal films coated on various types of substrates including glass, silicon, and polymer[11]. However, both of them are limited to fabricating periodic structures with simple shapes.

Here, we propose a rapid laser printing technology capable of fabricating both periodic and non-periodic micro- and nano-scale metallic structures using near field photothermal printing (NPTP) guided by a transparent phase-shifting mask. The schematic process using NPTP to fabricate periodic nanoparticles is illustrated in Fig 1. A laser pulse with uniform spatial light intensity passing through a PDMS phase-shifting mask redistributes its optical energy in space and forms a non-uniform light intensity profile at the interface of this PDMS mask and the underlying gold thin film. The laser pulses shaped by the PDMS phase-shifting mask selectively heat up and melt the gold film in areas with high light intensity. Due to instability of a molten gold film on a glass substrate, the molten film, driven by surface tension, quickly migrates to cold areas. The shape evolution of the molten film is controlled by surface tension of molten gold, laser pulse energy, duration, and pulse number. Since the formation of these gold nanostructures needs only one to few laser pulses, it has the potential for rapid, large-scale nanofabrication by scanning laser pulses across a large area. Furthermore, since the local shape of a PDMS mold determines the near field light pattern, NPTP has the potential for fabricating arbitrary shape of 2D metal nanostructures by designing the PDMS phase-shifting mask.

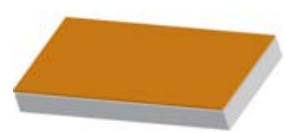

(a)

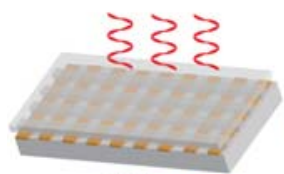

(d)

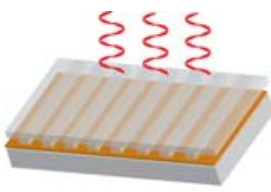

(b)

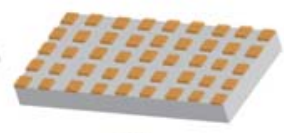

(e)

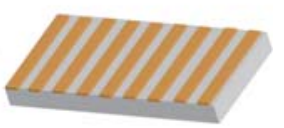

(c)

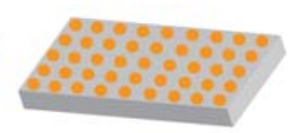

(f)
Figure 1: Schematic of NPTP processes. (a) A thin gold film is coated on a glass slide; (b) A 1-D grating PDMS mold is placed on top of a gold thin film and irradiated by pulsed laser; (c) Gold nanowires form after exposure; (d) Rotate the PDMS mold and repeat the above processes (b); (e) Isolated gold squares form; (f) Gold squares bead up and form nanospheres after flood laser exposure.

\section{SIMULATION AND EXPERIMENTAL RESULTS}

Fig. 2 shows the simulated light intensity distribution near a periodic PDMS mold and a gold thin film using the finite difference time domain (FDTD) method. The incident light is TE polarized with a wavelength of $532 \mathrm{~nm}$. Fig. 2(a) shows the grating pattern on the PDMS mold with a periodicity of $5 / 3 \mu \mathrm{m}$ and positioned in contact with a $10 \mathrm{~nm}$ gold film on a glass substrate. Fig 2(b) shows electric field redistribution during propagation through the grating structure. The incident uniform laser pulse is affected by the periodic PDMS grating structure, resulting in nonuniform profile. The total energy density distribution on the metal surface is plotted in Fig 2(c). The optical energy is strongly modified and shows a peak right underneath the grating tip and a valley between adjacent grating protuberances. Assume there is a threshold incident energy fluence for metal melting, by adjusting the pulse energy, gold thin film near the strong light intensity regions above the threshold is selectively melted while gold film near the low energy regions remain solid. Due to high surface tension of molten film, the molten gold migrates to the cold areas at a speed over $200 \mathrm{~m} / \mathrm{s}[12]$. After it quickly cools down, film rupture is expected to be observed. 


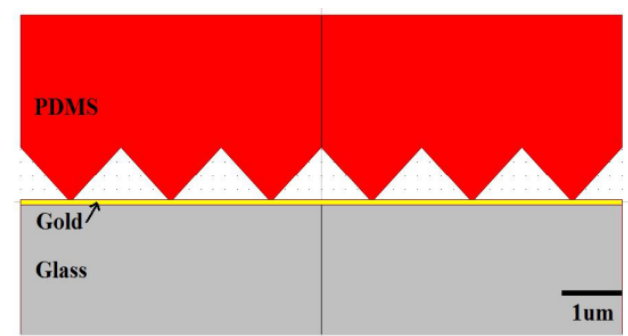

(a)

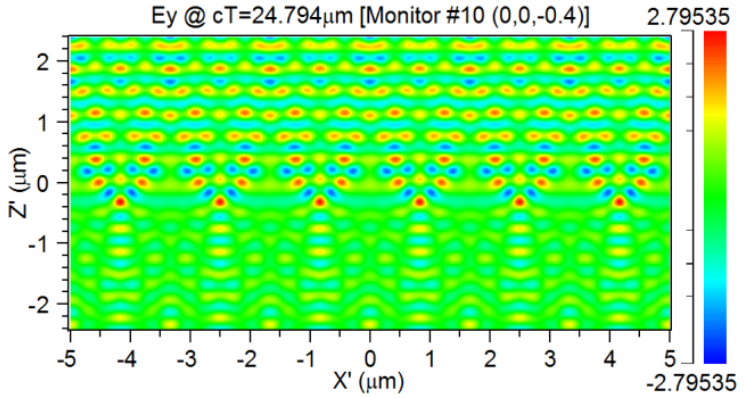

(b)

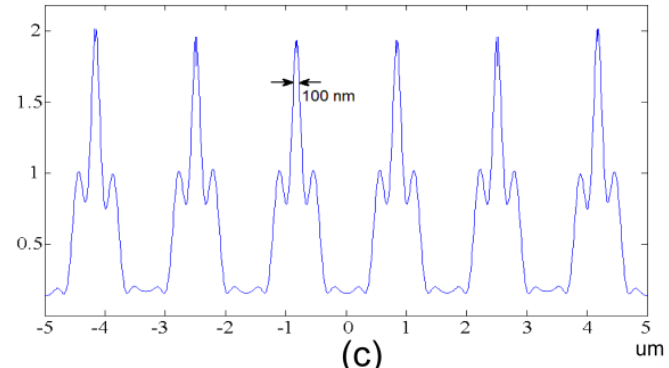

(c)

Figure 2: (a) PDMS/Au/Glass structure in Rsoft simulation; (b) FDTD Simulated electric field distribution during propagation through the grating structure; (c) Total energy density redistribution on the gold surface.

In the experiment, a Q-switched Nd:YAG pulse laser with a pulse width of $6 \mathrm{~ns}$ and a wavelength of $532 \mathrm{~nm}$ is applied. A $1 \mathrm{~nm}$ $\mathrm{Ti} / 10 \mathrm{~nm}$ gold thin film is deposited on a $100 \mu \mathrm{m}$ thick glass substrate by electron-beam evaporation. A PDMS mold with $5 / 3$ $\mu \mathrm{m}$ spacing grooves is simply a replica of a plane ruled reflection grating with $34^{\circ}$ nominal blaze angle and 600 grooves $/ \mathrm{mm}$ (10RG600-1850-2, Newport Corp).

To fabricate the PDMS phase-shifting mold, a $3 \mathrm{~mm}$ thick PDMS layer is first cured on top of a silicon wafer to provide a mechanical support for molding. A thin PDMS layer is then spin coated on the thick PDMS base. Before it is cured, the grating structure is placed on top of this thin PDMS film for molding. This process is carried out in vacuum to drive out the bubbles trapped between the grating and the PDMS. The PDMS mold is peeled off from the grating structure after it is cured.

Fig. 3 demonstrates the NPTP processes for fabricating a 1D gold nanowire array, 2D nanosquare and nanosphere arrays. During laser annealing process, the PDMS phase-shifting mold is generally positioned on the $\mathrm{Au}$ thin film without applying any external mechanical pressure. In Fig. 3(a), separated gold wires are formed after illuminating two laser pulses with a fluence of $80 \mathrm{~mJ} / \mathrm{cm}^{2}$. Smaller line width can be achieved by increasing the laser fluence such that a wider melting zone can be achieved. However, the laser fluence should also be kept smaller enough so that the peak intensity regions do not evaporate, which causes re-deposition of $\mathrm{Au}$ residues on the PDMS mold. The narrowing can also be achieved by increasing the pulse number. By rotating the PDMS mold by $90^{\circ}$ and followed by another three laser pulsing, gold nanowires are cut into squares (Fig 3(b)). More laser pulses or larger fluence is used in wire-cutting to achieve the same rupture due to the thicker nanowires compared to the initial continuous film. The shape of these isolated 2D nanosquares can evolve into 3D nanospheres by flood laser pulsing with a fluence of $150 \mathrm{~mJ} / \mathrm{cm}^{2}$. Molten metal squares ball up due to surface tension driven shape evolution and form nanospheres with smooth surface. Fig. 3(c) and 3(d) show the SEM images of two types of periodic gold nanospheres arrays. The latter formed by rotating the PDMS grating mold with $120^{\circ}$, with isolated diamonds formation before flood exposure. The periodicity of the nanospheres in two dimensions can also be different by using different grating periodicity in the two sequential exposures. A $6 \mathrm{~mJ}$ laser pulse utilized in this experiment is able to cover a print area of $1 \mathrm{~mm}^{2}$. Large print area can be accomplished by scanning the light beams across the phase shift mask.
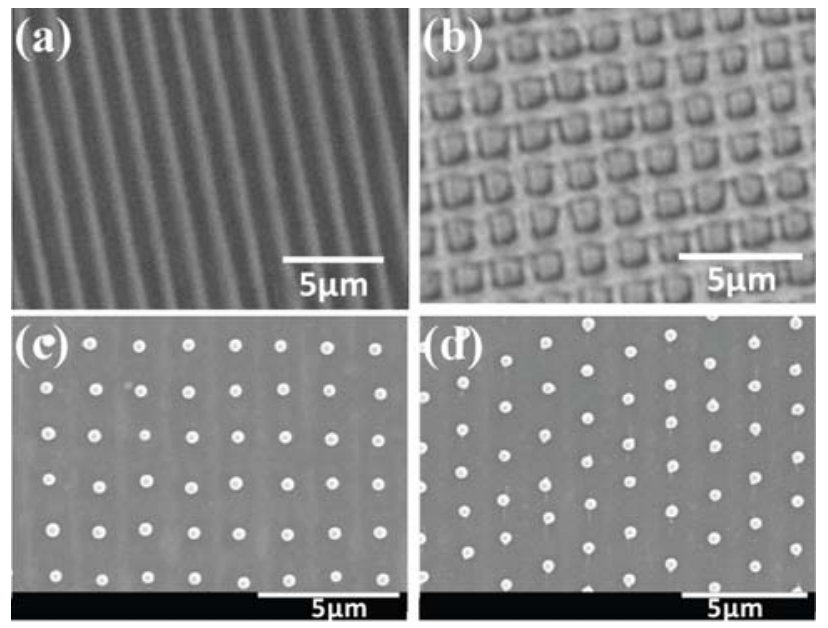

Figure 3: Photothermal annealed periodic nanostructures (lineline and particle-particle spacing is $5 / 3 \mu \mathrm{m}$ ). (a) gold nanowires; (b) $1 \times 1 \mathrm{\mu m}^{2}$ gold square before flood exposure; (c) $400 \mathrm{~nm}$ diameter gold nanospheres form orthogonal array after flood exposure; (d) hexagonal gold nanosphere array.

Since NPTP utilizes near field optical patterns to generate local heating to induce metal dewetting, the fabricated structures are not limited to periodic patterns. Any arbitrary shape of patterns can be fabricated by designing the phase shifting masks. Fig. 4 demonstrates photothermal annealing of few example patterns using NPTP. The PDMS phase shifting mold used here is designed by ourselves and fabricated by replicating a patterned photoresist master mold on a silicon wafer. All patterns in Fig. 4 are obtained by a single laser pulse. The metallic structure formed is determined by reconstructed optical energy distribution after passing through the phase-shifting mold, resulting in not exact one-to-one mapping compared to the phase-shifting pattern. By properly designing the PDMS phase-shift mask, holes, rings, triangles, crosses, squares, meshes, and more complex shape of structures can be achieved.

Plastic and polymer are two common types of materials used as flexible substrates in electronics and photonic applications. The 
melting temperature of these materials is usually much lower than the melting temperature of metals and semiconductors. Pulse laser annealing is a commonly applied technology in electronic industry to obtain high quality polycrystalline film since it minimizes heating to the underlining substrates and is compatible with the low thermal budget of these low melting temperature substrates. In NPTP, the pattern formation process takes place within $1 \mu \mathrm{s}$, so it also permits laser printing on substrates that have low thermal budget. Shown in Fig. 5 is NPTP printing of a 100nm thick gold thin film coated on a flexible PDMS substrate.
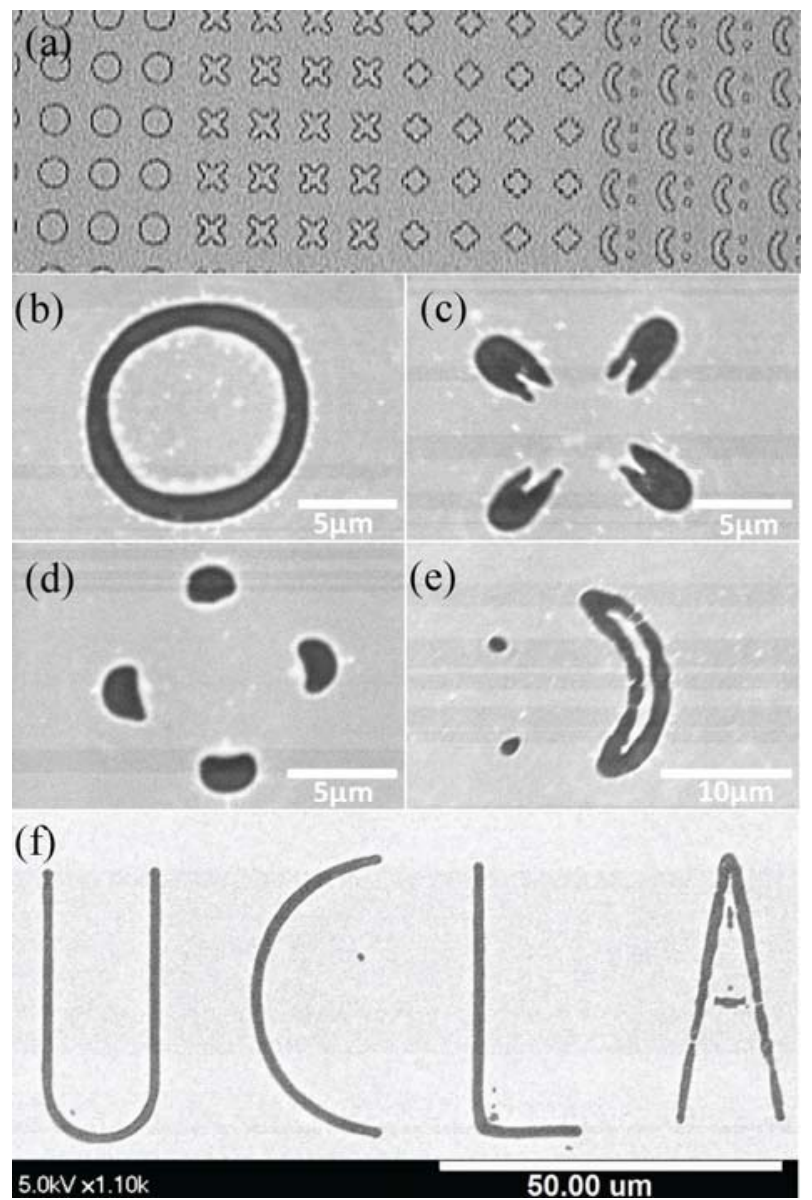

Figure 4: NPTA printing of arbitrary metallic structures (a) PDMS phase-shifting mask used to print arbitrary patterns; (b)-(e) arbitrary metallic patterns printed using NPTP corresponding to the phase-shifting patterns in (a); (f) "UCLA" pattern with smallest line width of $1 \mu \mathrm{m}$;

\section{DISCUSSION AND CONCLUSION}

The possible damage of PDMS phase-shifting mold is considered. One possible cause is contamination due to redeposition of evaporated metal. The laser pulse has a spatial Gaussian profile. In order to achieve large area fabrication, the maximum energy in the center of the laser spot can become high enough to evaporate the metal. By controlling the incident laser energy, the evaporation of metal can be avoided. The pulse laser annealing process takes place within $1 \mu \mathrm{s}$, so the burning of PDMS due to hot molten metal underneath is negligible.
The printing resolution of NPTP is mainly limited by two factors including near field optical field distribution resolution and the thermal diffusion length in the thin film. The former is controlled by the phase-shifting pattern on PDMS mold and incident laser energy fluence. Simulation shows that given the same periodicity, different phase-shifting pattern results in similar redistribution profile constituting of interlaced peaks and valleys, but in aspects of peak-valley ration and FWHM, the modulation of light still shows difference. By applying different energy fluence, the width above the melting threshold is different, which induces the primary melting of the metal film. From the simulation result shown in Fig. 2(c), a $100 \mathrm{~nm}$ wide optical heating line can be produced using near field phase shifting mold with a $532 \mathrm{~nm}$ wavelength light beam. However, this narrow heating area is broadened quickly in the lateral direction by thermal diffusion in the gold thin film during pulse duration $(6 \mathrm{nsec})$. The thermal diffusion length in a $10 \mathrm{~nm}$ thick gold film is $870 \mathrm{~nm}$ (estimated by $\mathrm{L}=(\mathrm{Dt})^{1 / 2}$ where $\mathrm{D}$ is the thermal diffusion coefficient on a $2 \mathrm{D}$ thin film and $\mathrm{t}$ is the pulse duration $6 \mathrm{nsec}$ ), which means the heat quickly diffuses to the adjacent area where the optical energy absorbed is under the melting threshold, and makes wider area melt. To increase the printing resolution of NPTP, laser pulses with shorted pulse duration can be used to reduce the thermal diffusion length.
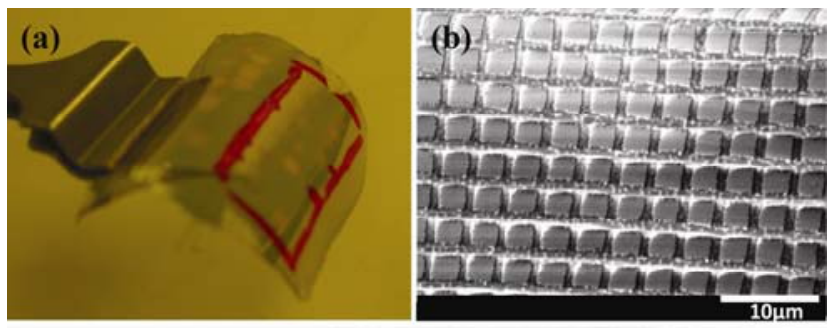

(c)

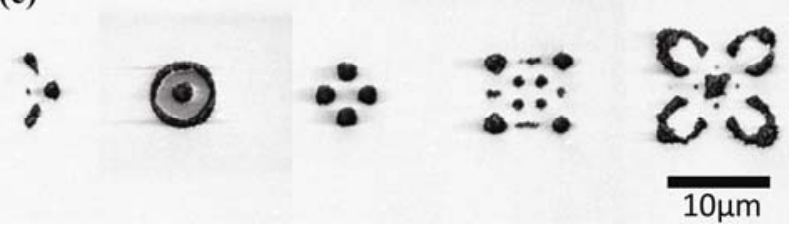

Figure 5: NPTA printing of metallic patterns on thin PDMS substrate; (a) PDMS substrate with thin gold layer on top of it; (b) Gold square array on PMDS; (c) Arbitrary metallic structures on PMDS.

To conclude, we have demonstrated a near field photothermal printing method for rapid laser printing of metallic micro and nanostructures, such as nanowires, nanosquares, and nanospheres and other arbitrary patterns. It utilizes laser induced dewetting and self-organization processes guided by spatially shaped light patterns between a phase shifting mask and the printed metal thin film. The rapid laser pulsing, metal dewetting, and shape evolution processes promise NPTP a high speed printing technology for fabricating metallic nanostructures.

\section{ACKNOWLEDGEMENT}

This project is supported in parts by the NSF grants CBET0853500 and NSF ECCS-0901154. 


\section{REFERENCE}

[1] Law, M., et al., Nanowire dye-sensitized solar cells. Nature materials, 2005. 4(6): p. 455-459.

[2] Righini, M., et al., Parallel and selective trapping in a patterned plasmonic landscape. Nature Physics, 2007. 3(7): p. 477-480.

[3] Laux, E., et al., Plasmonic photon sorters for spectral and polarimetric imaging. Nature Photonics, 2008. 2(3): p. 161164.

[4] $\mathrm{Wu}, \mathrm{T} . \mathrm{H}$., et al., Image patterned molecular delivery into live cells using gold particle coated substrates. Opt. Express, 2010. 18: p. 938-946.

[5] Cho, M.H., et al., Designed fabrication of multifunctional magnetic gold nanoshells and their application to magnetic resonance imaging and photothermal therapy. Angewandte Chemie (International ed. in English), 2006. 45(46): p. 2138344.

[6] Alivisatos, P., The use of nanocrystals in biological detection. Nature Biotechnology, 2004. 22(1): p. 47-52.

[7] Cui, B., C. Keimel, and S.Y. Chou, Ultrafast direct imprinting of nanostructures in metals by pulsed laser melting. Nanotechnology, 2010. 21: p. 045303.

[8] Xia, Q. and S.Y. Chou, The fabrication of periodic metal nanodot arrays through pulsed laser melting induced fragmentation of metal nanogratings. Nanotechnology, 2009. 20: p. 285310

[9] Kaganovskii, Y., H. Vladomirsky, and M. Rosenbluh, Periodic lines and holes produced in thin $\mathrm{Au}$ films by pulsed laser irradiation. Journal of Applied Physics, 2006. 100: p. 044317.

[10] Nishioka, K. and S. Horita, Periodic arrays of submicron Si and $\mathrm{Ni}$ dots on $\mathrm{SiO} 2$ fabricated using linearly polarized $\mathrm{Nd}$ : YAG pulsed laser. Applied Physics A: Materials Science \& Processing, 2008. 91(2): p. 235-240.

[11] Moon, J.H., J. Ford, and S. Yang, Fabricating threedimensional polymeric photonic structures by multi-beam interference lithography. Polymers for Advanced Technologies, 2006. 17(2): p. 83-93.

[12] Bischof, J., et al., Dewetting modes of thin metallic films: Nucleation of holes and spinodal dewetting. Physical review letters, 1996. 77(8): p. 1536-1539.

\section{CONTACT}

*Fan Xiao, tel: +1-310-880-1399; michille521@gmail.com 